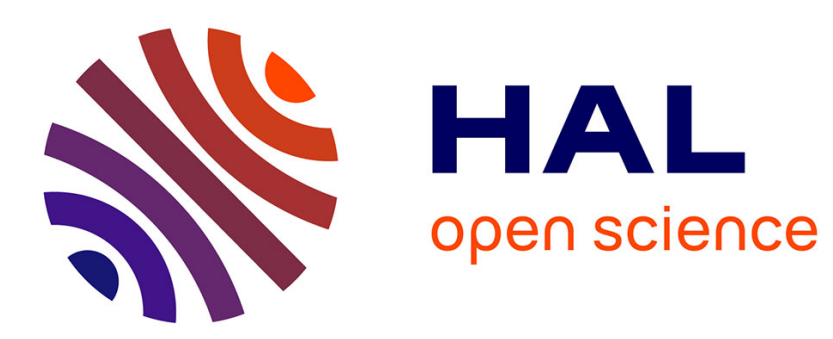

\title{
Tunable fall velocity of a dense ball in oscillatory cross-sheared concentrated suspensions
}

\author{
Frédéric Blanc, Elisabeth Lemaire, François Peters
}

\section{To cite this version:}

Frédéric Blanc, Elisabeth Lemaire, François Peters. Tunable fall velocity of a dense ball in oscillatory cross-sheared concentrated suspensions. Journal of Fluid Mechanics, 2014, 746, pp.R4. hal-01026306

\section{HAL Id: hal-01026306 https://hal.science/hal-01026306}

Submitted on 21 Jul 2014

HAL is a multi-disciplinary open access archive for the deposit and dissemination of scientific research documents, whether they are published or not. The documents may come from teaching and research institutions in France or abroad, or from public or private research centers.
L'archive ouverte pluridisciplinaire HAL, est destinée au dépôt et à la diffusion de documents scientifiques de niveau recherche, publiés ou non, émanant des établissements d'enseignement et de recherche français ou étrangers, des laboratoires publics ou privés. 


\title{
Tunable fall velocity of a dense ball in oscillatory cross-sheared concentrated suspensions
}

\author{
Frédéric Blanc, Elisabeth Lemaire and François Peters $\dagger$ \\ CNRS, Université de Nice, LPMC-UMR 7336, 06108 Nice Cedex 2, France
}

(Received 20 March 2014)

The fall velocity of a dense large ball in a suspension of neutrally-buoyant nonBrownian particles submitted to horizontal oscillatory shear is studied. As the strain amplitude is increased, the velocity increases up to a maximum value before it decreases to the value that it would have in a resting suspension. The higher the frequency, the stronger the effect. The falling ball velocity can be largely increased in the presence of the oscillatory cross-shear flow. For instance, it reaches for a particle volume fraction $\Phi=0.47$ four times the value it has in the unsheared suspension. At small strain amplitudes, it turns out that the falling ball velocity is determined by a balance between the steady drag flow, which drives the apparent suspension viscosity toward a high value, and the oscillatory cross-shear that lessens it. A simple model is proposed to explain the experimental observations at small strain amplitude. The velocity decrease observed at larger amplitude is not completely understood yet.

Key words:

\section{Introduction}

Suspensions display a wide range of rheological properties which are closely connected to the so-called shear-induced microstructure (Morris 2009). In the field of suspensions of non-colloidal particles in a Newtonian liquid, the pair distribution function that quantifies the microstructure has been measured experimentally (Parsi \& Gadala-Maria 1987; Rampall et al. 1997; Gao et al. 2010; Cheng et al. 2011; Blanc et al. 2011a, 2013) and computed in simulations (Sierou \& Brady 2002; Yeo \& Maxey 2010). It basically shows a strongly enhanced pair probability at contact in the compression quadrant of the ambiant flow. It is known to originate in the non-hydrodynamic forces between particles and to result in a large viscosity increase together with the development of anisotropic normal stresses (Sierou \& Brady 2002; Boyer et al. 2011; Dbouk et al. 2013). For example, in a shear reversal experiment (Gadala-Maria \& Acrivos 1980; Kolli et al. 2002; Narumi et al. 2002; Blanc et al. 2011b), where the microstructure is destroyed and then built again by the shear flow, the viscosity can be reduced by a factor ten or more, depending on the particle volume fraction, as the microstructure vanishes (Bricker \& Butler 2007) before it increases again to its steady value. Such a suspension, if submitted to an oscillatory shear flow, displays a complex behavior that has been studied by several authors (Breedveld et al. 2001; Pine et al. 2005; Bricker \& Butler 2006; Corte et al. 2008; Park et al. 2011; Lin et al. 2013). The viscosity at small strain amplitude is found to be much smaller

† Email address for correspondence: Francois.Peters@unice.fr 
than the steady shear viscosity. Again, the strong variation of the rheological properties with the strain amplitude reflects a qualitative change of the microstructure (Bricker \& Butler 2007), and the time evolution seems to originate in the mechanical irreversibility presumably due to contact forces between particles (Pine et al. 2005; Corte et al. 2008; Lin et al. 2013).

In the present paper, we focus on the slow sedimentation of a dense large ball in a concentrated suspension of neutrally buoyant non-colloidal particles in a Newtonian liquid. In an unbounded purely Newtonian liquid, the drag on the sphere is well known (Stokes 1851; Happel \& Brenner 1983) and the sphere velocity is simply given as a function of the liquid viscosity $\eta$, the density of the particle $\rho_{p}$, of the liquid, $\rho_{s}$, the particle radius $a_{f}$ and the gravitational acceleration $g$ :

$$
v=\frac{2}{9} \frac{\left(\rho_{p}-\rho_{s}\right) a_{f}^{2} g}{\eta}
$$

If the vessel boundaries influence has to be taken into account, Eq.1.1 yields an apparent viscosity that can be corrected depending on the vessel shape and the sphere position (Happel \& Brenner 1983). The falling motion of a dense ball in concentrated suspensions has been the subject of several studies (Mondy et al. 1986; Reardon et al. 2007). If the ball is large enough compared to the size of the particles in the suspension, and once the boundary effects are corrected, the apparent viscosity is close to the value that is measured from steady-shear rheometry. This suggests that the shear rate around the falling ball induces a microstructure in the suspension that is oriented by the local strain rate axis and that determines the viscosity felt by the ball.

Here, we present experimental evidence that the falling ball velocity increases significantly when the suspension is subjected to a uniform horizontal oscillatory shear rate, especially at low strain amplitude and high oscillation frequency, whereas the dynamic viscosity of the suspension remains roughly constant.

\section{Experiments}

\subsection{Materials and experimental device}

The experiments are performed in a wide-gap Couette cell made of PMMA (figure 1) mounted on a controlled stress rheometer Mars II (Haake, Thermofisher). The cylindrical cup of radius $R_{o}=24 \mathrm{~mm}$ is machined into a parallelepipedic block $(106 \mathrm{~mm}$ in height, $60 \times 60 \mathrm{~mm}^{2}$ in cross-section) and the rotor is a cylinder of radius $R_{i}=14$ $\mathrm{mm}$. Transparent suspensions are made of monodisperse spherical PMMA particles (Microbeads CA30), $2 a_{s}=31 \pm 4 \mu m$ in diameter, dispersed in a Newtonian fluid (viscosity $\eta_{0}=1.02 \mathrm{~Pa} . \mathrm{s}$ ) that has been designed (Cargille Laboratories) to match the density and refractive index of the particles. Two suspensions of particle volume fraction $\Phi=0.40$ and 0.47 are studied. Dense balls are dropped along the centerline of the gap. Their final position at the bottom of the cup is always within $a_{f} / 2$ of the gap center. In the following, we thus assume a constant radial position of the ball, i.e $r=r_{c}=19 \mathrm{~mm}$. We use two types of ball: stainless steel balls (specific gravity $7760 \pm 80 \mathrm{~kg} . \mathrm{m}^{-3}$ and $2 a_{f}=1.0$ $\mathrm{mm} \pm 10 \mu \mathrm{m}$ in diameter) and aluminium balls (specific gravity $2900 \pm 30 \mathrm{~kg} \cdot \mathrm{m}^{-3}$ and $2 a_{f}=1.0 \mathrm{~mm} \pm 10 \mu \mathrm{m}$, Goodfellow). An externally triggered camera (Basler acA2000$340 \mathrm{~km}$ ) records images of the falling ball (figure 1). Each frame (2048 $p x \times 1088 p x$ in size) is processed to extract the ball size and position (Blanc et al. 2011a, 2013). The apparent radius of the ball is $11 p x$ and the accuracy in the ball center position is \pm 0.1 $p x$. Since the radial position of the falling ball is constant, its vertical coordinate can be 


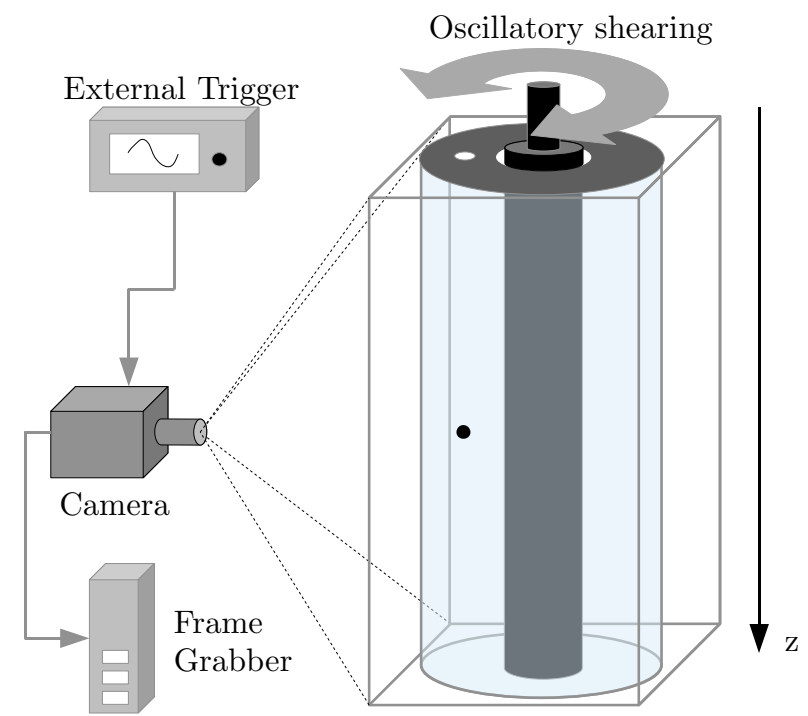

Figure 1: Experimental device.

computed from its position in the frame after a necessary calibration. In order to validate the experimental set-up, steel balls are dropped in a Newtonian oil. The apparent viscosity from Eq.1.1 is approximately 10\% larger than the otherwise measured viscosity. This is consistent with the influence of the cylinder walls as modeled by two infinite vertical planes $10 \mathrm{~mm}$ apart (Happel \& Brenner 1983). No influence of the oscillatory shear rate is observed.

\subsection{Rheological characterization}

Local viscosity measurements in the wide-gap Couette cell (Blanc et al. 2011c) show a slightly shear thinning behavior of the suspensions in the range of shear rate applied in this study, namely $10^{-3} \mathrm{~s}^{-1} \lesssim \dot{\gamma} \lesssim 1 \mathrm{~s}^{-1}$. The viscosity at $\Phi=0.40$ (resp. 0.47) and $\dot{\gamma} \sim 0.1 \mathrm{~s}^{-1}$ is $\eta_{s}=12.8$ Pa.s (resp. 58.9 Pa.s) and the constitutive law is $\sigma \sim \dot{\gamma}^{0.975}$ (resp. $\left.\sigma \sim \dot{\gamma}^{0.875}\right)$.

Dynamic rheological measurements performed either using the wide-gap rheometer at the same time as the falling ball experiments (figure 2a) or in a parallel-plate torsional flow (figure $2 \mathrm{~b}$ ) are in good agreement. Since the out-of phase-viscosity is always quite small, only the magnitude of the complex dynamic viscosity $\left|\eta^{*}\right|$ is considered in the following. It does not vary much in the strain amplitude range of interest $\left(10^{-4} \lesssim \gamma_{0} \lesssim\right.$ 1 ) and it does not depend on frequency at all in the range $(0.2 \mathrm{~Hz} \leqslant f \leqslant 20 \mathrm{~Hz})$. As a consequence, the strain amplitude $\gamma_{0}$ at the middle of the gap is computed from the angular amplitude of the rotor assuming a Newtonian behavior of the suspension. The time evolution of the viscosity deserves a few comments: after a very short transient (one or two cycles) following the onset of the oscillations, $\left|\eta^{*}\right|$ goes to an initial value that is much smaller than the steady shear viscosity at $\dot{\gamma} \sim 0.1 \mathrm{~s}^{-1}$. Then it changes slowly, but the variations do not exceed $5 \%$ of the initial viscosity for $\Phi=0.40$ (resp. $10 \%$ for $\Phi=0.47$ ). This behavior is in qualitative agreement with previously published short term viscosity measurements (Breedveld et al. 2001; Park et al. 2011; Lin et al. 2013). The low dynamic viscosity has been attributed to the development of a particular shear-induced microstructure (Corte et al. 2008; Lin et al. 2013). 

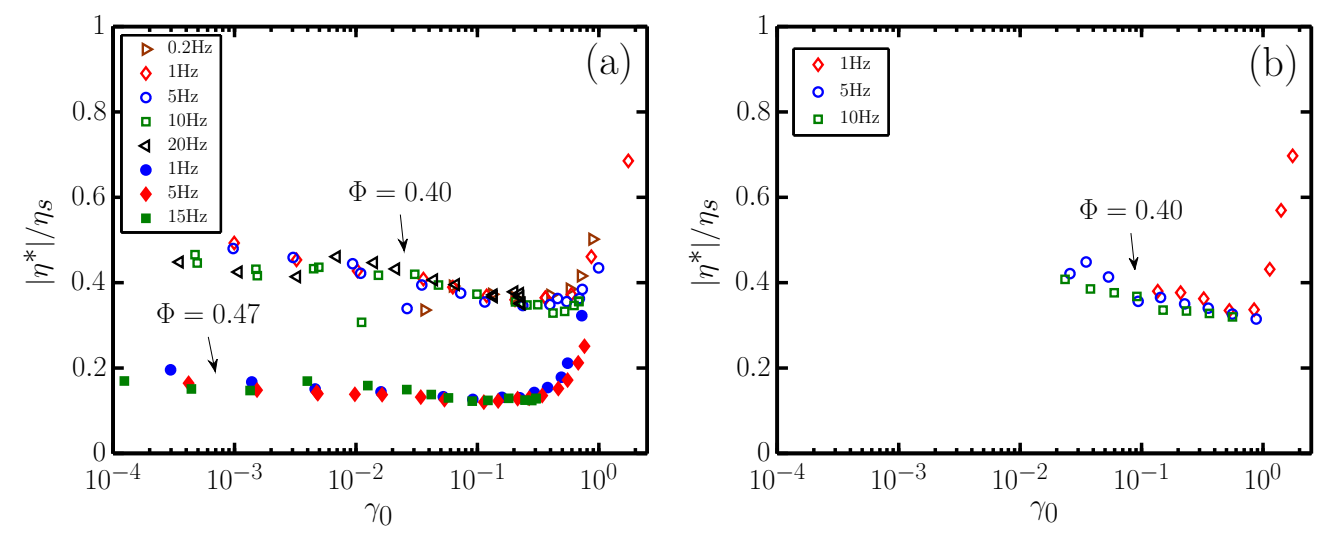

Figure 2: Dynamic viscosity $\left|\eta^{*}\right|$ normalized by the steady viscosity at $\dot{\gamma} \sim 0.1 \mathrm{~s}^{-1}$ against the strain amplitude for different frequencies of strain oscillation. Measurements performed : $(a)$ in the wide-gap Couette cell $(\Phi=0.40$ and 0.47$) ;(b)$ in a parallel-plate geometry $(\Phi=0.40)$.

\section{Results}

\subsection{Falling motion in suspensions at rest}

When a ball is dropped in a resting suspension, the velocity undergoes the same type of transient as in the Newtonian oil (figure 3), as already reported (Reardon et al. 2007), before it reaches a steady value $v_{0}$. The apparent viscosity from Eq.1.1 is within 10\% of that is measured using a rheometer at the same steady shear rate as estimated by $\dot{\gamma}_{\text {sed }}=v_{0} / a_{f} \sim 0.6 \mathrm{~s}^{-1}$. Since $\left(R_{o}-R_{i}\right) / a_{f}=20$, the bounding wall effect is expected to be the same as in a Newtonian liquid (Mondy et al. 1986). An important point is that this steady velocity depends only slightly on the initial suspension microstructure. The same measurement performed after the suspension was oscillatory presheared (figure 3) yields a steady velocity only $5 \%$ (resp. $10 \%$ ) larger at $\Phi=0.40$ (resp. $\Phi=0.47$ ), whereas the dynamic viscosity during oscillation is approximately 0.35 (resp. 0.12) times the viscosity in steady shear flow. At last, even though we did not perform extensive measurements, the falling ball velocity seems to be the same whether the measurement is done when the suspension is continuously sheared or at rest. This observation has been made for a steady shear rate equal to $\dot{\gamma}=0.35 \mathrm{~s}^{-1}$, i.e. $\dot{\gamma} a_{f} / v_{0} \sim 0.6$, in the $\Phi=0.40$ suspension. All these observations hold also for the falling motion of the aluminium balls. It confirms that the microstructure induced by the sphere motion itself is mostly responsible for the high viscosity felt by the sphere in steady motion.

\subsection{Falling motion in oscillatory cross-sheared suspensions}

An oscillatory torque is now applied and the ball vertical position $z$, together with the shear strain amplitude $\gamma_{0}$, is measured one time per cycle. In all reported experiments, the inertia of the suspension and of the falling ball can be neglected. Figure 3 displays the falling ball velocity as oscillation alternates with rest periods. As the oscillation is applied, the sphere velocity increases during typically two cycles to a nearly constant value, $v$, while the viscosity probed by the rheometer reaches a steady value, shown in figure 2 . As the oscillation is stopped, the velocity decreases to its initial value $v_{0}$. The normalized fall velocity $v / v_{0}$ for the suspension with volume fraction $\Phi=0.40$ is displayed in figure $4 \mathrm{a}$ for steel and aluminium balls as a function of the strain amplitude $\gamma_{0}$ for different values of the frequency. Each point corresponds to the falling ball velocity averaged over 


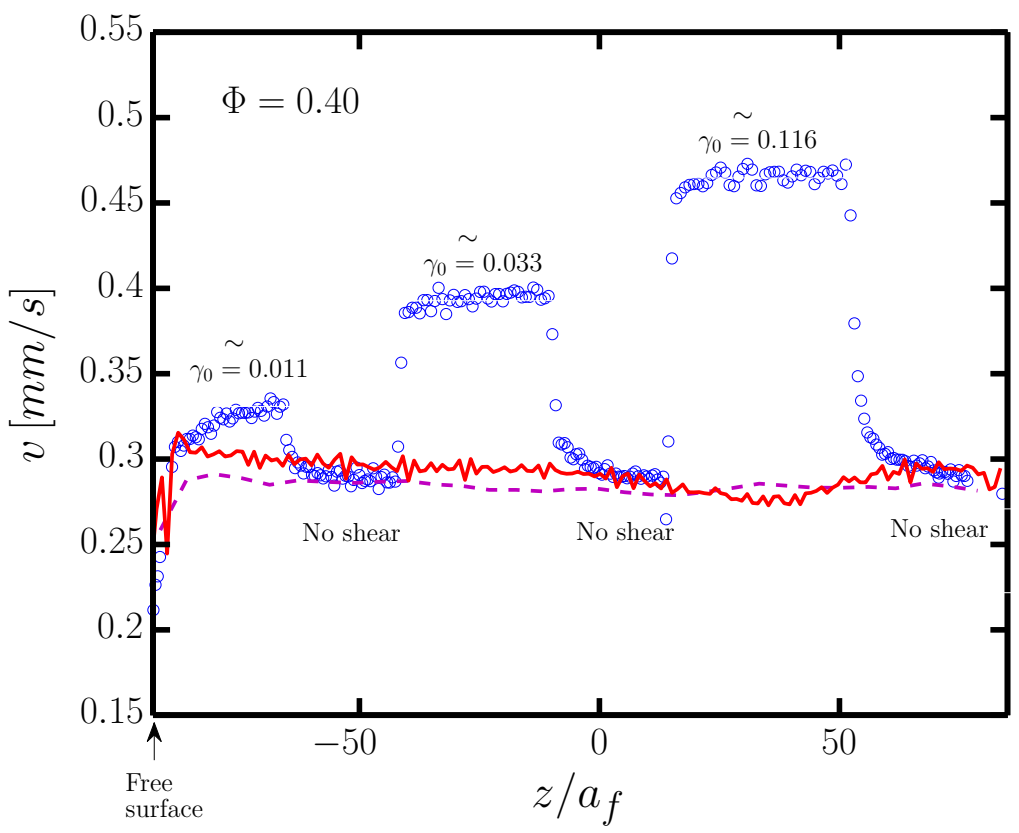

Figure 3: Fall velocity of a steel ball as a function of vertical position. $\Phi=0.40$. $(--)$ Velocity in suspension at rest after steady preshearing [shear rate $\sim 0.1 \mathrm{~s}^{-1}$ during 60 $\mathrm{s}] ;(-)$ Velocity in suspension at rest after the same steady preshearing followed by 6000 cycles of oscillation $\left(f=5 \mathrm{~Hz}, \gamma_{0} \simeq 0.33\right)$; (o) Velocity during a sequence of oscillations $(f=5 \mathrm{~Hz})$ interspersed with rest times.
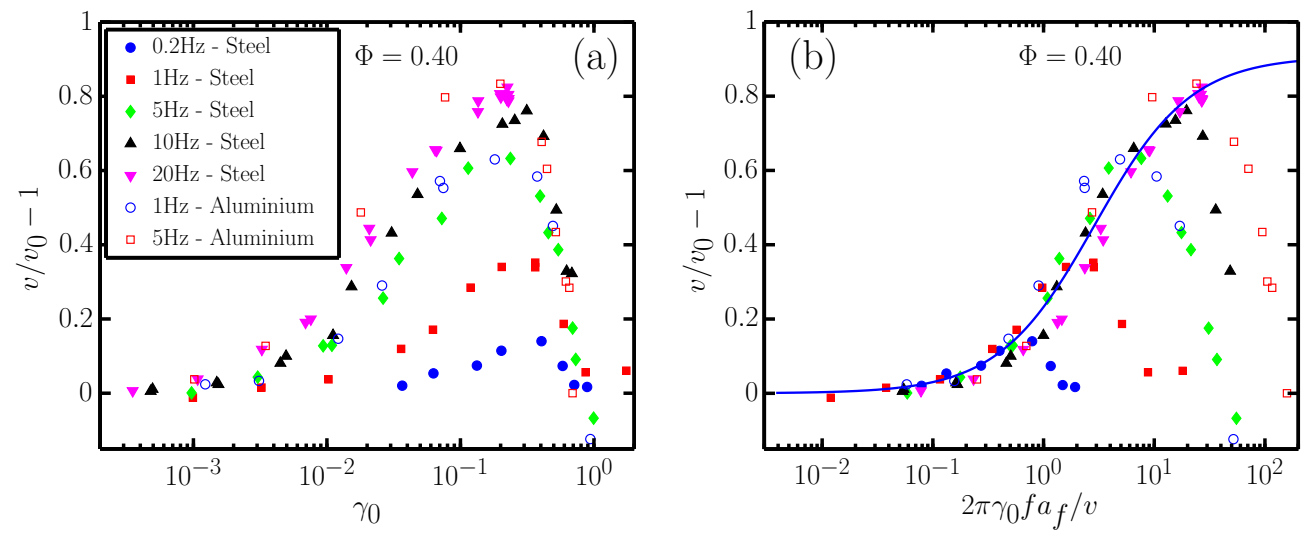

Figure 4: (a) Relative velocity increase as a function of strain amplitude. (b) Relative velocity increase as a function of reduced shear rate; same legend as in (a); solid line : model. $\Phi=0.40$.

the plateau after transient (figure 3), resulting in a standard deviation that roughly corresponds to the size of the symbols. As expected from Eq.1.1 $v_{0 \text { Steel }} / v_{0 \text { Alu }} \approx 4$. The striking feature is here that the velocity largely depends on the frequency and the strain amplitude of the external shear flow, whereas the suspension viscosity measured by the rheometer hardly changes with both parameters. The same results are displayed as a 

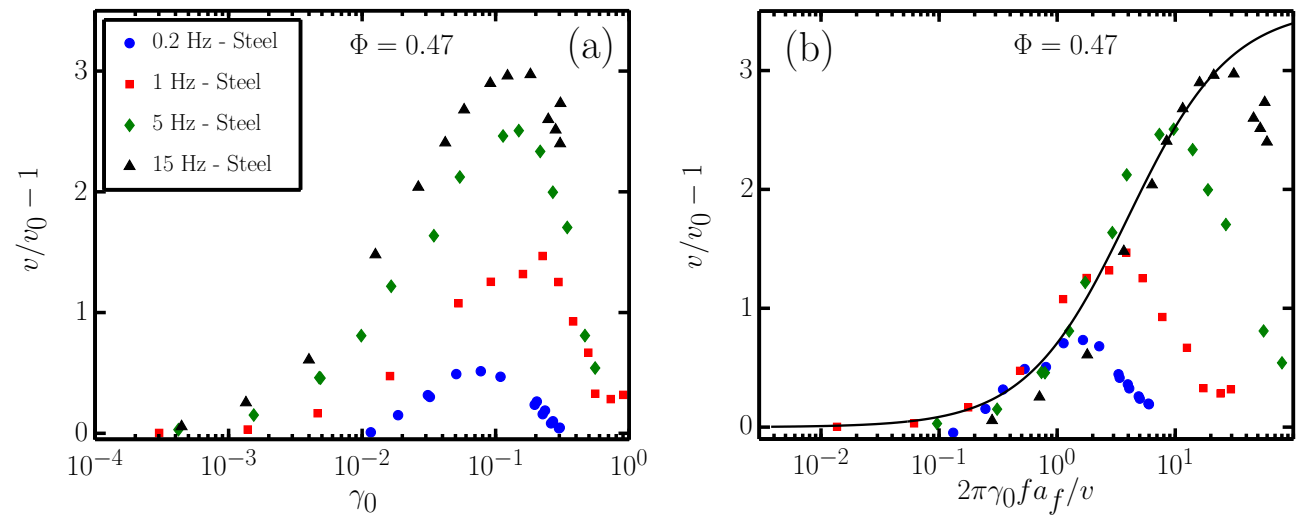

Figure 5: (a) Relative velocity increase as a function of strain amplitude. (b) Relative velocity increase as a function of reduced shear rate; same legend as in (a). Solid line: model. $\Phi=0.47$.

function of the ratio of the imposed shear rate $\dot{\gamma}=2 \pi \gamma_{0} f$ to the shear rate induced by the falling ball motion $v / a_{f}$ (figure $4 \mathrm{~b}$ ). With this scaling, all data collapse onto one single curve at small strain amplitude. In that range, the velocity seems to be determined by the competition between the falling motion of the sphere that builds the usual microstructure around it, and the external oscillatory shear rate that would destroy it. However, as the strain amplitude increases beyond typically $\gamma_{M} \sim 0.2-0.3$, the former scaling does not hold anymore. The velocity decreases and reaches at a strain amplitude around $\gamma_{m} \sim 0.9$ the value that it would have in a resting suspension. This strong decrease cannot be explained by the quite weak variation of the dynamic viscosity with the strain amplitude (figure 2). The same qualitative features are evidenced in the $\Phi=0.47$ suspension with $\gamma_{M} \sim 0.15$ and $\gamma_{m} \sim 0.6$ (figure 5 ).

\section{Model and discussion}

To get a deeper insight into the small amplitude behavior, we build a simple model, where the apparent viscosity obeys a relaxation equation that involves two competing mechanisms:

$$
\frac{d \eta_{a p p}}{d t}=-\frac{v}{a_{f}} \frac{\eta_{a p p}-\eta_{\max }}{\gamma_{c 1}}-\frac{2 \pi f \gamma_{0}}{\gamma_{c 2}}\left(\eta_{a p p}-\eta_{\text {min }}\right)
$$

where $v$ and $v_{0}$ are respectively related to $\eta_{a p p}$ and $\eta_{\max }$ through $\eta_{a p p} / \eta_{\max }=v_{0} / v$ (Eq.1.1). The first term is related to the building of the suspension microstructure around the falling ball that drives the apparent viscosity towards the value $\eta_{\max }$, which is deduced from the velocity $v_{0}$ in the resting suspension. In analogy with shear reversal experiments (Gadala-Maria \& Acrivos 1980; Kolli et al. 2002; Narumi et al. 2002; Blanc et al. 2011b), where the viscosity is controlled by the total strain experienced by the suspension, a characteristic strain $\gamma_{c 1}$ together with the typical shear rate around the falling ball $v / a_{f}$ determines the relaxation rate (see supplementary material). The characteristic relaxation length $z_{c}=a_{f} \gamma_{c 1}$ is roughly estimated from the transient velocity after the oscillation is stopped (figure 3 ). The characteristic strain $\gamma_{c 1} \approx 3.5$ (resp. 2.3) at $\Phi=0.40$ (resp. $\Phi=0.47$ ) is in qualitative agreement with shear reversal experiments with the same suspension (Blanc et al. 2011b, and supplementary material). The second relaxation term of Eq.4.1 drives the apparent viscosity to the lower value $\eta_{\min }$. The cor- 
responding relaxation rate is taken proportional to the imposed shear rate $2 \pi f \gamma_{0}$ that is responsible for the modification of the microstructure. The ratio $\gamma_{c 2} / \gamma_{c 1}$, together with $\eta_{\min } / \eta_{\max }$, is deduced from the best fit of the experimental measurement to the steady solution of Eq.4.1 (figures $4 \mathrm{~b} \& 5 \mathrm{~b}$ ). $\gamma_{c 2}$ is equal to 5.3 (resp. 2.2) at $\Phi=0.40$ (resp. $\Phi=0.47) . \gamma_{c 1}$ and $\gamma_{c 2}$ are of the same order of magnitude, suggesting that both building and destruction of the microstructure are driven by the strain experienced by the suspension around the sphere. The ratio $\eta_{\min } / \eta_{\max }$ is the inverse of the larger relative velocity $v / v_{0}$ that the model could predict. As expected, the computed values $\left(\eta_{\min } / \eta_{\max }=0.52\right.$ at $\Phi=0.40$ and $\eta_{\min } / \eta_{\max }=0.22$ at $\left.\Phi=0.47\right)$ are quite close to the relative dynamic viscosity shown in figure 2 . As shown in supplementary material, the parameters found above allow us to compute the transient response at the inception and the end of the oscillations, and the agreement is quite satisfactory.

While this simple model seems to catch the mechanisms that control the falling ball velocity at low strain amplitude, it is unable to explain the decrease of the velocity as the amplitude grows further. The decrease of the falling ball velocity seems to be controlled by the strain amplitude: for a given volume fraction, the velocity reaches its maximum value at approximately the same strain amplitude, $\gamma_{M}$, whatever the frequency, and decreases to $v_{0}$ for approximately the same amplitude $\gamma_{m}$. This behavior deserves a few comments. First, even though the viscosity measured using the rheometer does not vary in a large extent over the amplitude range of interest, one cannot but notice that the increase of dynamic viscosity occurs from approximately $\gamma_{M}$ (figure 2). In addition, this typical strain amplitude determines approximately the minimum of the viscosity in a shear reversal experiment performed with the same suspension (Blanc et al. 2011b, and supplementary material). This set of observations suggests that the effect of the imposed shear on the suspension microstructure changes qualitatively as the strain amplitude exceeds $\gamma_{M}$. In recent papers, such a qualitative change was evidenced (Pine et al. 2005; Corte et al. 2008), i.e. the development of long term irreversibility and diffusion for strain amplitudes larger than a critical value $\gamma_{c}$. Below $\gamma_{c}$, oscillations drive the system into a so-called absorbing state where the particles do not longer undergo mutual collisions. The time necessary to reach this state is an increasing function of the amplitude and seems to diverge at $\gamma_{c}$ (Corte et al. 2008). In recent rheological experiments (Lin et al. 2013) the short term evolution of the rheological properties toward a steady oscillatory state showed the same slowing down, which was attributed to the same origin. Lin et al. (2013) found $\gamma_{c}=1.22$ at $\Phi=0.40$ and $\gamma_{c}=0.67$ at $\Phi=0.5$, which is close to the values that we measure for the strain amplitude $\gamma_{m}$ necessary for the velocity to decrease to $v_{0}$, namely $\gamma_{m} \sim 0.9$ (resp. 0.6) at $\Phi=0.40$ (resp. $\Phi=0.47$ ). In addition, the amplitude where the transient time measured by Lin et al. (2013) starts increasing compares well with $\gamma_{M}$ in our experiment. Even though, in our case, the suspension around the ball cannot reach the absorbing state, since it is constantly structured by the drag flow induced by the falling motion, the decrease of the velocity above $\gamma_{M}$ could be explained by the weaker efficiency of the external shear flow in breaking the structure, akin to the increasing time necessary to drive the suspension toward a steady oscillatory behavior.

\section{Conclusion}

In this paper, we have shown that it is possible to increase significantly the fall velocity of a large dense ball in a concentrated suspension by applying an oscillatory smallamplitude horizontal cross-shear strain. The higher the particle volume fraction, the greater the effect. The falling motion of the dense ball can not be predicted by the sus- 
pension dynamic viscosity alone since the latter hardly changes with strain amplitude and frequency. Apparently, the motion of the ball changes the microstructure in its vicinity, resulting in an apparent viscosity that strongly depends on the oscillatory cross-shear flow characteristics. At small amplitude, the ball velocity increase is determined by the competition between the falling motion of the sphere that builds the usual microstructure around it, and the external oscillatory shear rate that breaks it. The velocity decrease that occurs at larger amplitude is not completely understood yet and seems to be connected to the increasing time that is necessary for the particles to rearrange under the influence of the oscillatory shear (Corte et al. 2008; Lin et al. 2013).

More generally, this study adresses the issue of the suspension response to the superposition of two orthogonal shear rates. This has proven fruitfull in the field of viscoelastic fluids (Van den Brule \& Gheissary 1993; Padhy et al. 2013) or particle suspensions in a yield-stress fluid (Ovarlez et al. 2012). While this issue has been rarely considered in the field of particle suspensions in Newtonian liquids (Barral 2011; Hanotin et al. 2012), it provides an opportunity to probe the influence of the microstructure as it is not simply imposed by a steady simple shear rate, but can be tuned using the oscillatory shear flow. In our experiments, the actual flow around the ball is probably complicated, and we think that further experiments in a more controlled rheometric cross-shear flow would provide valuable information on the connection between microstructure and rheology.

The possibility to tune the settling velocity of a non-neutrally buoyant falling ball in suspensions should have some applications in problems involving suspension degassing. Such an effect might also be involved in soil liquefaction during earthquakes.

The authors gratefully acknowledge Laurent Lobry for fruitfull discussions.

\section{REFERENCES}

BARral, Quentin 2011 Superposition d'écoulements orthogonaux dans des fluides complexes: mise en place de l'expérience, application aux suspensions et aux fluides à seuil. $\mathrm{PhD}$ thesis, Université Paris-Est.

Blanc, F, Lemaire, E, Meunier, A \& Peters, F 2013 Microstructure in sheared nonbrownian concentrated suspensions. Journal of Rheology $\mathbf{5 7}$ (1), 273-292.

Blanc, Frédéric, Peters, François \& Lemaire, Elisabeth 2011a Experimental signature of the pair trajectories of rough spheres in the shear-induced microstructure in noncolloidal suspensions. Physical Review Letters 107 (20), 208302.

Blanc, F, Peters, F \& Lemaire, E $2011 b$ Local transient rheological behavior of concentrated suspensions. Journal of Rheology $\mathbf{5 5}$ (4), 835-854.

Blanc, Frédéric, Peters, François, Lemaire, Elisabeth et al. 2011c Particle image velocimetry in concentrated suspensions: Application to local rheometry. Applied Rheology 21, 23735.

Boyer, François, Guazzelli, Élisabeth \& Pouliquen, Olivier 2011 Unifying suspension and granular rheology. Physical Review Letters 107, 188301.

Breedveld, Victor, van den Ende, Dirk, JongschaAp, Robert \& Mellema, Jorrit 2001 Shear-induced diffusion and rheology of noncolloidal suspensions: Time scales and particle displacements. The Journal of Chemical Physics 114 (13), 5923-5936.

BRICKER, JM \& ButleR, JE 2006 Oscillatory shear of suspensions of noncolloidal particles. Journal of Rheology $\mathbf{5 0}$ (5), 711-728.

Bricker, JonATHAN M \& Butler, JASON E 2007 Correlation between stresses and microstructure in concentrated suspensions of non-brownian spheres subject to unsteady shear flows. Journal of Rheology $\mathbf{5 1}$ (4), 735-759.

VAn Den Brule, BHAA \& Gheissary, G 1993 Effects of fluid elasticity on the static and dynamic settling of a spherical particle. Journal of Non-Newtonian Fluid Mechanics 49 (1), 123-132. 
Cheng, Xiang, McCoy, Jonathan H, Israelachvili, Jacob N \& Cohen, Itai 2011 Imaging the microscopic structure of shear thinning and thickening colloidal suspensions. Science 333 (6047), 1276-1279.

Corte, Laurent, Chaikin, PM, Gollub, JP \& Pine, DJ 2008 Random organization in periodically driven systems. Nature Physics 4 (5), 420-424.

Dbouk, T., Lobry, L. \& Lemaire, E. 2013 Normal stresses in concentrated non-brownian suspensions. Journal of Fluid Mechanics 715, 239-272.

Gadala-Maria, F \& Acrivos, Andreas 1980 Shear-induced structure in a concentrated suspension of solid spheres. Journal of Rheology 24 (6), 799-814.

Gao, C, Kulkarni, SD, Morris, JF \& Gilchrist, JF 2010 Direct investigation of anisotropic suspension structure in pressure-driven flow. Physical Review E 81 (4), 041403.

Hanotin, Caroline, de Richter, S Kiesgen, Marchal, Philippe, Michot, Laurent J \& Baravian, Christophe 2012 Vibration-induced liquefaction of granular suspensions. Physical Review Letters 108 (19), 198301.

HAPPEL, J \& BRENNER, H 1983 Low reynolds number hydrodynamics .

Kolli, Venkata Giri, Pollauf, Emily J \& Gadala-Maria, Francis 2002 Transient normal stress response in a concentrated suspension of spherical particles. Journal of Rheology 46 (1), 321-334.

Lin, Yuan, Phan-Thien, Nhan \& Khoo, Boo Cheong 2013 Short-term and long-term irreversibility in particle suspensions undergoing small and large amplitude oscillatory stress. Journal of Rheology $5 \mathbf{7}$ (5), 1325-1346.

Mondy, LA, Graham, AL \& Jensen, JL 1986 Continuum approximations and particle interactions in concentrated suspensions. Journal of Rheology 30 (5), 1031-1052.

Morris, JefFrey F 2009 A review of microstructure in concentrated suspensions and its implications for rheology and bulk flow. Rheologica Acta 48 (8), 909-923.

Narumi, Takatsune, See, Howard, Honma, Yuichi, Hasegawa, Tomilchi, Takahashi, Tsutomu \& Phan-Thien, Nhan 2002 Transient response of concentrated suspensions after shear reversal. Journal of Rheology 46 (1), 295-305.

Ovarlez, Gulllaume, Bertrand, François, Coussot, Philippe \& Chateau, Xavier 2012 Shear-induced sedimentation in yield stress fluids. Journal of Non-Newtonian Fluid Mechanics 177, 19-28.

Padhy, S, Shaqfeh, ESG, Iaccarino, G, Morris, JF \& Tonmukayakul, N 2013 Simulations of a sphere sedimenting in a viscoelastic fluid with cross shear flow. Journal of Non-Newtonian Fluid Mechanics 197, 48-60.

Park, Hyun-OK, Bricker, Jonathan M, Roy, Michael J \& Butler, Jason E 2011 Rheology of oscillating suspensions of noncolloidal spheres at small and large accumulated strains. Physics of Fluids 23, 013302.

PARsi, F \& Gadala-Maria, F 1987 Fore-and-aft asymmetry in a concentrated suspension of solid spheres. Journal of Rheology 31 (8), 725-732.

Pine, DJ, Gollub, JP, Brady, JF \& Leshansky, AM 2005 Chaos and threshold for irreversibility in sheared suspensions. Nature 438 (7070), 997-1000.

RAmpall, IndResh, SMART, JEFFREY R \& Leighton, DAVID T 1997 The influence of surface roughness on the particle-pair distribution function of dilute suspensions of non-colloidal spheres in simple shear flow. Journal of Fluid Mechanics 339, 1-24.

Reardon, Patrick T, Graham, Alan L, Feng, Shihai, Chawla, Vibha, Admuthe, RAHul S \& Mondy, LisA A 2007 Non-newtonian end effects in falling ball viscometry of concentrated suspensions. Rheologica Acta 46 (3), 413-424.

Sierou, A \& BRADY, JF 2002 Rheology and microstructure in concentrated noncolloidal suspensions. Journal of Rheology 46 (5), 1031-1056.

Stokes, GG 1851 On the effect of the internal friction of fluids on the motion of pendulums. Transactions of the Cambridge Philosophical Society $\mathbf{9}, 8$.

Yeo, Kyongmin \& MaXey, Martin R 2010 Dynamics of concentrated suspensions of noncolloidal particles in couette flow. Journal of Fluid Mechanics 649 (1), 205-231. 